\title{
Double blind, controlled trial of 4-aminosalicylic acid and prednisolone enemas in distal ulcerative colitis
}

\author{
L J D O'Donnell, A S Arvind, P Hoang, D Cameron, I C Talbot, D P Jewell,
} J E Lennard-Jones, M J G Farthing

\begin{abstract}
Corticosteroid or 5-aminosalicylic acid enemas are the treatment of choice for distal ulcerative colitis but up to one third of patients may be unresponsive. As an alternative therapy might be advantageous, the efficacy of six weeks' treatment with 2 g 4-aminosalicylic acid (4-ASA) $(n=24)$ and $20 \mathrm{mg}$ prednisolone enemas $(n=21)$ were compared in a double blind, randomised trial in patients with acute distal ( $<30 \mathrm{~cm}$ from the anus) ulcerative colitis. Baseline demography and clinical severity were similar in both groups. Five of 24 patients receiving 4-ASA and 4 of 21 receiving prednisolone did not complete the trial because of deteriorating symptoms, failure to improve, or side effects. At the time of leaving the trial, 24 hour stool frequency, the presence of blood in the stools, and histological and sigmoidoscopic appearances were similar in both groups. Symptomatic improvement occurred in 17 of 24 patients receiving 4-ASA compared with 11 of 21 receiving prednisolone $\left(\chi^{2}=1.62\right.$, NS). Complete symptomatic improvement occurred in 9 of 24 patients receiving 4-ASA compared with 5 of 21 receiving prednisolone $\left(\chi^{2}=0.98, N S\right)$. Histological improvement was seen in 9 of 24 patients on 4-ASA compared with 7 of 21 on prednisolone $\left(\chi^{2}=0.08\right.$, NS). One patient receiving 4-ASA was considered to have an idiosyncratic reaction to the drug but other side effects were not considered to be drug related. Thus, 4-ASA, previously used in the treatment of tuberculosis (para-aminosalicylic acid), is as good as prednisolone in the treatment of distal ulcerative colitis and should be considered in patients unresponsive to steroids or in whom steroid treatment is undesirable.
\end{abstract}

Corticosteroid enemas are of proved efficacy for the topical treatment of distal ulcerative colitis. However, as treatment failures may occur in up to $30 \%$ of patients ${ }^{1}$ and as up to $40 \%$ of some rectally administered corticosteroids are absorbed systemically, ${ }^{23}$ alternative treatments are desirable. 5-aminosalicylic acid (ASA), which is the active component of sulphasalazine ${ }^{+}$ has been shown to be as good as, if not better, than corticosteroids in the treatment of mild to moderate left sided ulcerative colitis when given as an enema. ${ }^{5}$ However, as with steroids, failures also occur with 5-ASA enemas and alternative treatments have been sought.

4-ASA is an isomer of 5-ASA. This drug, otherwise known as para-aminosalicylic acid, has been used since the 1940s in the treatment of tuberculosis. Its safety and low toxicity have been well documented. ${ }^{6}$ In double blind placebo controlled trials, 4-ASA enemas have been shown to be safe and effective in the treatment of distal ulcerative colitis. $^{7-9}$ To establish further the clinical usefulness of 4-ASA given as an enema we have compared its efficacy with prednisolone enemas, an established therapy for the treatment of ulcerative colitis. ${ }^{10}$

\section{Methods}

Fifty three patients with distal ulcerative colitis recruited from gastroenterology outpatient clinics were randomly assigned to receive either 4-ASA (aminosalicylic sodium USP $2 \mathrm{~g}$, lactose $1.995 \mathrm{~g}$, colloidal silicon dioxide $5 \mathrm{mg}$ ) or prednisolone (prednisolone sodium phosphate USP $20 \mathrm{mg}$, lactose $1.995 \mathrm{~g}$, colloidal silicon dioxide $5 \mathrm{mg}$ ) enemas. Both enemas were made up with $50 \mathrm{ml}$ of tap water just before rectal instillation at bedtime. All patients gave written informed consent and the study was approved by the Ethical Committees of the City and Hackney Health Authority, London and the Oxfordshire Health Authority. Patients were entered into the study following establishment of a histological diagnosis of ulcerative colitis with active disease confined to $30 \mathrm{~cm}$ or less of the distal colon as judged by rigid sigmoidoscopy. If rigid sigmoidoscopy did not show an upper limit of disease, barium enema or colonoscopy was used to confirm that disease was confined to the distal $30 \mathrm{~cm}$ of the rectum and colon. Patients were excluded if enteropathogens were detected on microscopy or culture of faeces, if there was a history of allergy to salicylates, inability to retain an enema for greater than 30 minutes, pregnancy or lactation, or use of steroid enemas, antibiotics or non-steroidal anti-inflammatory drugs in the week before entry. Patients taking oral forms of sulphasalazine, mesalazine, or corticosteroids were included only if the dose of these drugs had been stable for at least three months before entry.

At the start of the six week trial period all patients had a full clinical assessment and the following symptoms were recorded and scored as either absent (1), mild (2), moderate (3), or severe (4): blood in stools, urgency, tenesmus, mucus, abdominal and/or rectal pain, fatigue, weight loss, fever, and malaise. Stool frequency and consistency (firm $=1$, semi-formed $=2$, or loose $=3$ ) were also recorded. Sigmoidoscopic appearances were recorded $(0=$ normal, $1=$ loss of vascular pattern, $2=$ contact bleeding, 
$3=$ discrete ulceration, and/or spontaneous bleeding) and a rectal biopsy specimen was obtained. The patients' view of well-being was scored on a visual analogue scale, from 0 (as bad as possible) to 100 (as good as possible). Patients were issued with a six week supply of enemas and instructed to retain each enema overnight.

Throughout the trial, patients were asked to keep a daily record of their symptoms and were seen at two week intervals for follow up. At the final visit another rectal biopsy specimen was obtained. All clinical, histological, and symptomatic assessments by patients and investigators were performed double blind.

Histological assessment of the rectal biopsy specimens was performed blind by a consultant histopathologist (ICT) and scored as follows: $0=$ normal, $1=$ mild colitis (infiltrate minimally exceeding normal lymphoplasmacytic background), 2 =moderate colitis (infiltrate expands lamina propria and leukocytes enter crypt or surface epithelium without crypt abscess or ulceration), and $3=$ severe colitis (intense infiltrate with crypt abscess or surface ulceration).

Baseline characteristics in the two groups were compared using a two tailed $t$ test. The within treatment changes from baseline were tested by a one tailed, paired $t$ test. The treatments were compared at the post-baseline visits using analysis of covariance; the treatment group was used as the factor and the baseline value as the covariant. The $\chi^{2}$ test was also used to compare the two treatment groups at the time of trial exit.

\section{Results}

Of the 53 patients initially randomised to the trial, five were excluded from analysis because of failure to adhere to the trial protocol (two on prednisolone, three on 4-ASA) and three were excluded as the extent of their disease was later shown to be $>30 \mathrm{~cm}$ (one patient) or else a diagnosis of ulcerative colitis was not confirmed (two patients); all three were randomised to receive prednisolone. Baseline characteristics of the remaining 45 patients in the two treatment groups are shown in the Table. There was no statistically significant difference between the two groups.

Thirty seven of the 45 patients completed the six week trial. Five of the 24 patients receiving 4-ASA failed to complete the trial because of deteriorating symptoms (two patients), side effects (one patient), or failure to improve and unwillingness to continue (two patients). Three of the 21 prednisolone patients failed to complete the trial because of deteriorating symptoms (two patients), or failure to improve and unwillingness to continue (one patient).

The data were analysed at the time of trial exit for each patient; that is at the end of six weeks for those completing the trial and at the time of withdrawal for those who withdrew early. In one patient (receiving 4-ASA enemas) trial exit data were missing. At the time of trial exit, blood in stools and sigmoidoscopic and histological appearances had improved significantly from baseline with both treatments (Table). However, at the time of trial exit stool frequency showed a significant improvement only in those patients receiving 4-ASA.

Other assessment scores at the time of trial exit are also shown in the Table. Fever, malaise, pain, fatigue, and weight loss were not particularly severe at baseline hence there was no significant improvement in these parameters in either group during the trial. However, patients receiving 4-ASA enemas had improvements in tenesmus, urgency, passage of mucus rectally, and stool consistency whereas those receiving prednisolone only had improvement in passage of mucus (Table). There were no significant differences between the two treatment groups in any of the assessment scores at the time of trial exit.

Symptomatic improvement in at least one symptom occurred in 17 of 24 patients on 4-ASA compared with 11 of 21 taking prednisolone $\left(\chi^{2}=\right.$ $1 \cdot 62$, NS). Complete symptomatic improvement, defined as absence of urgency and rectal bleeding and bowel frequency of less than twice a

Effects of 4-aminosalicylic acid and prednisolone enemas in distal ulcerative colitis

\begin{tabular}{|c|c|c|c|c|c|}
\hline \multirow{3}{*}{$\begin{array}{l}\text { Age (years) (mean }(\mathrm{SD})) \\
\text { Sex } \\
\text { Weight }(\mathrm{kg})(\text { mean }(\mathrm{SD})) \\
\text { Patients on mesalazine or sulphasalazine }\end{array}$} & \multicolumn{2}{|c|}{ 4- $A S A(n=24)$} & \multicolumn{3}{|c|}{ Prednisolone $(n=21)$} \\
\hline & \multicolumn{2}{|c|}{$\begin{array}{l}49(18) \\
14 \mathrm{~F} \\
65(9 \cdot 3) \\
\quad 18\end{array}$} & \multicolumn{2}{|c|}{$\begin{array}{l}43(16) \\
13 F \\
66(14) \\
15\end{array}$} & \multirow[b]{2}{*}{$\begin{array}{l}\text { Difference } \\
(95 \% C I) \text { at exit }\end{array}$} \\
\hline & $\begin{array}{l}\text { Baseline } \\
\text { mean }(S D)\end{array}$ & $\begin{array}{l}\text { Trial exit } \\
\text { mean }(S D)\end{array}$ & $\begin{array}{l}\text { Baseline } \\
\text { mean }(S D)\end{array}$ & $\begin{array}{l}\text { Trial exit } \\
\text { mean }(S D)\end{array}$ & \\
\hline $\begin{array}{l}\text { Blood in stools }(1-4) \\
24 \text { hour stool frequency } \\
\text { Stool consistency }(1-3) \\
\text { Urgency }(1-4) \\
\text { Tenesmus }(1-4) \\
\text { Mucus }(1-4) \\
\text { Rectal or abdominal pain }(1-4) \\
\text { Weight loss }(1-4) \\
\text { Malaise }(1-4) \\
\text { Fatigue }(1-4) \\
\text { Fever }(1-4) \\
\text { Patients view of well-being }(0-100) \\
\text { Sigmoidoscopic appearances }(0-3) \\
\text { Histological appearances }(0-3)\end{array}$ & $\begin{array}{l}2 \cdot 6(0 \cdot 7) \\
4 \cdot 5(3 \cdot 2) \\
2 \cdot 1(0 \cdot 8) \\
2 \cdot 5(0 \cdot 8) \\
2 \cdot 0(0 \cdot 8) \\
2 \cdot 4(0 \cdot 7) \\
1 \cdot 8(0 \cdot 8) \\
1 \cdot 1(0 \cdot 3) \\
1 \cdot 2(0 \cdot 4) \\
1 \cdot 6(0 \cdot 7) \\
1 \cdot 1(0 \cdot 6) \\
67 \cdot 0(16) \\
2 \cdot 7(0 \cdot 5) \\
2 \cdot 0(1 \cdot 1)\end{array}$ & 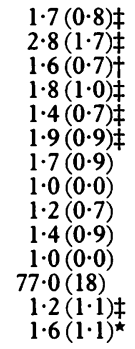 & $\begin{array}{l}2 \cdot 5(0 \cdot 8) \\
3 \cdot 4(2 \cdot 0) \\
1 \cdot 8(0 \cdot 7) \\
2 \cdot 4(1 \cdot 0) \\
2 \cdot 0(0 \cdot 8) \\
2 \cdot 5(0 \cdot 7) \\
1 \cdot 6(0 \cdot 6) \\
1 \cdot 2(0 \cdot 7) \\
1 \cdot 6(0 \cdot 8) \\
1 \cdot 6(0 \cdot 9) \\
1 \cdot 1(0 \cdot 4) \\
69 \cdot 0(16) \\
2 \cdot 3(0 \cdot 7) \\
2 \cdot 2(0 \cdot 9)\end{array}$ & 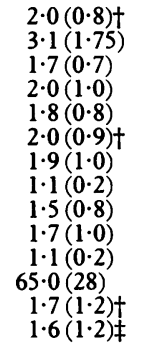 & $\begin{array}{l}-0.28(-0.78 \text { to } 0.22) \\
-0.55(-1.59 \text { to } 0.48) \\
-0.12(-0.56 \text { to } 0.32) \\
-0.21(-0.83 \text { to } 0.40) \\
-0.41(-0.90 \text { to } 0.07) \\
-0.05(-0.6 \text { to } 0.51) \\
-0.38(-0.91 \text { to } 0.16) \\
-0.05(-0.14 \text { to } 0.05) \\
-0.29(-0.78 \text { to } 0.19) \\
-0.34(-0.85 \text { to } 0.17) \\
\\
12.45(-0.64 \text { to } 25.5) \\
-0.47(-1.23 \text { to } 0.29) \\
0.14(-0.54 \text { to } 0.81)\end{array}$ \\
\hline
\end{tabular}

See methods section for description of assessment scores. 4-ASA and prednisolone groups were not significantly different in any of the baseline characteristics. Significance of differences from baseline $\star<0.05, t<0.02, \ddagger<0.01$. There were no significant differences between the two treatment groups at the time of trial exit. Exit differences were estimated using analysis of covariance, taking baseline differences into consideration. 
day, occurred in 9 of 24 patients receiving 4-ASA compared to 5 of 21 receiving prednisolone $\left(\chi^{2}=\right.$ $0.98, \mathrm{NS}$ ).

Change in patients' view of well-being, from baseline to trial exit assessed by a visual analogue scale $(0=$ as bad as possible, $100=$ as good as possible) improved from a mean of 67 to 77 in the 4-ASA group and deteriorated slightly from 69 to 65 in the prednisolone group. Sixteen of the 4-ASA patients view of well being improved during the trial compared to 12 of the prednisolone patients $\left(\chi^{2}=0.43, \mathrm{NS}\right)$.

Histological improvement was seen in 9 of 24 patients on 4-ASA compared with 7 of 21 on prednisolone $\left(\chi^{2}=0.08, N S\right)$. Histological remission occurred in five patients on 4-ASA and five on prednisolone $\left(\chi^{2}=0.06, \mathrm{NS}\right)$.

To exclude the possibility that this trial was conducted to a significant extent in patients unresponsive to steroids, we performed further analysis omitting patients who were previously receiving steroid enemas for their current exacerbation. There were eight of these patients - four randomised to receive prednisolone and four to receive 4-ASA enemas. In the remaining patients at the time of trial exit there was no significant difference between the 4-ASA and prednisolone groups in stool frequency $(2.62 v 3.01$; difference $-0.39,95 \% \mathrm{CI}-1.64$ to 0.86 ), blood in stools ( 1.74 v 1.99 ; difference $-0.25,95 \% \mathrm{CI}-0.86$ to $0.34)$ sigmoidoscopic appearance $(1.22 v 1.46$; difference $-0 \cdot 24,95 \% \mathrm{CI}-1 \cdot 11$ to 0.62 ) or histological score $(1.65 v 1.35$; difference 0.30 , $95 \%$ CI -0.48 to $1 \cdot 08)$. Similarly, there was no significant difference between the two subgroups in any of the other end points (data not shown).

One patient receiving 4-ASA enemas developed malaise, nausea, and fever within two days of starting the drug and was considered to have an idiosyncratic reaction. One other patient taking 4-ASA developed self limiting headaches during the trial but this was thought unlikely to be caused by the trial medication. Two patients taking prednisolone complained of minor side effects (headache, abdominal pain) which were considered not to be related to the trial medication.

\section{Discussion}

This study shows that 4-ASA enemas are an effective treatment for distal ulcerative colitis. Previous studies have shown that 4-ASA enemas are superior to placebo, ${ }^{7-9}$ and the present study that 4-ASA enemas are as effective as prednisolone enemas in distal ulcerative colitis. In many of the subjective and objective criteria assessed in the trial, 4-ASA showed a tendency to be better than prednisolone but this did not achieve statistical significance. It is also clear from this trial that 4-ASA exerts its initial beneficial effects predominantly in controlling the symptoms of distal colitis and that histological improvement lags behind symptomatic improvement. Recent reports have indicated that oral administration of 4-ASA can help maintain remission of ulcerative colitis." These findings along with our data indicate that 4-ASA may have a wider role to play in the treatment of this condition.

In clinical practice 5-ASA enemas are the most commonly used alternative to prednisolone enemas in the treatment of distal colitis. Campieri et al have shown that 4-ASA enemas are as effective as 5-ASA enemas in this condition. ${ }^{12}$ Thus, 4-ASA in an addition to the armamentarium used in the treatment of ulcerative colitis and in view of its relative inexpensiveness, should prove advantageous.

In conclusion, $2 \mathrm{~g}$ 4-ASA enemas given for six weeks at night are an effective treatment of distal ulcerative colitis and should prove a welcome alternative to corticosteroid enemas.

This study was supported by a Research grant by Reed and Carnrick/Stafford-Miller Ltd. MJGF gratefully acknowledges financial support by the Wellcome Trust.

1 Truelove SC. Treatment of ulcerative colitis with local hydrocortisone hemisuccinate sodium. A report on a controlled therapeutic trial. $B M \mathcal{F}$ 1958; ii: $1072-7$.

2 Lee DAH, Taylor M, James WHT, Walker G. Plasma prednisolone levels and adrenocortical responsiveness after administration of prednisolone 21-phosphate as retension enema. Gut 1979; 20: 349-55.

3 Powell-Tuck J, Lennard-Jones JE, May CS, Wilson CG, Paterson JW. Plasma prednisolone levels after administration of prednisolone 21-phosphate as a retention enema in colitis. BMF 1976; i: 193-5.

4 Azad Khan AK, Piris J, Truelove SC. An experiment to determine the active therapeutic moiety of sulphasalazine. determine the active
Lancet 1977 ; ii: 892-5.

5 Campieri M, Lanfranchi GA, Bazzocchi G, et al. Treatment of ulcerative colitis with high dose 5-aminosalicylic acid enemas. Lancet 1981; ii: 270-1

6 Reynolds EF, ed. Martindale. The Extra Pharmacopeia. London: The Pharmaceutical Press, 1989.

7 Selby WS, Bennet, MK, Jewell DP. Topical treatment of distal ulcerative colitis with 4-aminosalicylic acid enemas. Digestion 1984; 29: 231-4.

8 Gandolfo J, Farthing M, Powers G, et al . 4-aminosalicylic acid retention enemas in treatment of distal colitis. Dig Dis $S c$ 1987; 32: 700-4.

9 Ginsberg AL, Beck LS, McIntosh TM, Nochomovitz LE. Treatment of left-sided ulcerative colitis with 4aminosalicylic acid enemas. A double-blind, placebo conaminosalicylic acid enemas. A double-blind,
trolled trial. Ann Intern Med 1988; 108: 195-9.

10 Matts SGF. Local treatment of ulcerative colitis with prednisolone-21-phosphate enema. Lancet 1960; i: 517-9.

11 Ginsberg AL, Davis ND, Nochomovitz LE. Placebo controlled double blind trial of oral 4-aminosalicylic acid in ulcerative colipis. Gastroenterology 1990; 98: A 172 .

12 Campieri M, Lanfranchi GA, Bertoni $F$, et al. A double-blind clinical trial to compare the effects of 4-aminosalicylic acid to 5 -aminosalicylic acid in topical treatment of ulcerative colitis. Digestion 1984; 29: 204-8. 